\title{
NOTE ON THE RELATION BETWEEN CONTINUITY AND DEGREE OF POLYNOMIAL APPROXI- MATION IN THE COMPLEX DOMAIN*
}

BY J. L. WALSH AND W. E. SEWELL

1. Introduction. It is the purpose of the present note to establish the following theorems:

THEOREM I. Let $C$ be an analytic Jordan curve in the z-plane and let $f(z)$ be defined in $\bar{C}$, the closed limited point set bounded by $C$. For each $n, n=1,2, \cdots$, let a polynomial $P_{n}(z)$ of degree $n$ in $z$ exist such that

$$
\left|f(z)-P_{n}(z)\right| \leqq \frac{M}{n^{p+\alpha}}, \quad z \text { in } \bar{C}, \quad 0<\alpha \leqq 1,
$$

where $M$ is a constant independent of $n$ and $z$, and $p$ is a nonnegative integer. Then $f(z)$ is analytic in $C$ and continuous in $\bar{C}$; the pth derivative $f^{(p)}(z)$ exists on $C$ in the one-dimensional sense and satisfies the condition

$$
\begin{array}{r}
\left|f^{(p)}\left(z_{1}\right)-f^{(p)}\left(z_{2}\right)\right| \leqq L\left|z_{1}-z_{2}\right| \alpha|\log | z_{1}-z_{2}|| \beta, \\
z_{1}, z_{2} \text { on } C,
\end{array}
$$

where $\beta=0$ if $\alpha<1$, and $\beta=1$ if $\alpha=1$, and where $L$ is a constant independent of $z_{1}$ and $z_{2}$.

TheOREM II. Let $E$, with boundary $C$, be a closed limited point set in the z-plane whose complement $K$ is connected, and is regular in the sense that there exists a function $w=\phi(z)$ which maps $K$ conformally but not necessarily uniformly onto $|w|>1$ so that the points at infinity in the two planes correspond to each other. Let the locus $C_{R}:|\phi(z)|=R>1$, consist of a finite number of mutually exterior analytic Jordan curves. Let $f(z)$ be defined in $E$, and for each $n, n=1,2, \cdots$, let a polynomial $P_{n}(z)$ of degree $n$ in $z$ exist such that

$$
\left|f(z)-P_{n}(z)\right| \leqq \frac{M}{n^{p+\alpha+1} R^{n}}, \quad z \text { in } E, \quad 0<\alpha \leqq 1,
$$

\footnotetext{
* Presented to the Society, March 27, 1937.
} 
where $M$ is a constant independent of $n$ and $z$, and $p$ is a nonnegative integer. Then $f(z)$ when suitably defined exterior to $E$ is analytic in $C_{R}$ and continuous in $\bar{C}_{R}$; the pth derivative $f^{(p)}(\boldsymbol{z})$ exists on $C_{R}$ in the one dimensional sense and satisfies the condition

$$
\begin{array}{r}
\left|f^{(p)}\left(z_{1}\right)-f^{(p)}\left(z_{2}\right)\right| \leqq L\left|z_{1}-z_{2}\right|^{\alpha}|\log | z_{1}-z_{2}||^{\beta}, \\
z_{1}, z_{2} \text { on } C_{R},
\end{array}
$$

where $\beta=0$ if $\alpha<1$, and $\beta=1$ if $\alpha=1$, and $L$ is a constant independent of $z_{1}$ and $z_{2}$.

In these theorems the case $p=0$ is not excluded:

$$
f^{(0)}(z) \equiv f(z) .
$$

Sewell* has already proved the slightly less general results that under the hypotheses of Theorems I and II the function $f^{(p)}(z)$ satisfies a Lipschitz condition $\dagger$ of every order $\alpha^{\prime}<\alpha$ on $C$ and $C_{R}$, respectively.

J. Curtiss has shown $\ddagger$ that if the boundary $C$ of a closed limited point set $E$ consists of a finite number of mutually exterior analytic Jordan curves, if $f(z)$ is analytic in the interior points of $E$ and continuous in $E$, and if $f^{(p)}(z)$ exists in the onedimensional sense on $C$ and satisfies on $C$ a Lipschitz condition of order $\alpha, 0<\alpha \leqq 1$, then there exist polynomials $P_{n}(z)$ of respective degrees $n$ such that (1) is valid for $z$ in $E$ with $\beta=0$ even for $\alpha=1$. Thus Theorem I is for $0<\alpha<1$ an exact converse of Curtiss's result. We show by an example that for $\alpha=1$ an exact converse is impossible.

To be sure, Curtiss did not state his result in the above form, but assumed $f^{(p)}(z)$ continuous in $E$. Nevertheless the lighter assumption is sufficient for his purposes, because we prove below (Theorem III) that this lighter assumption implies also the heavier assumption.

In the notation of Theorem II suppose $\left|f(z)-P_{n}(z)\right| \leqq \epsilon_{n}$, $z$ on $E, n=1,2, \cdots$, where $\epsilon_{n}$ approaches zero as $n$ becomes infinite. The study of the relation between $\epsilon_{n}$ and the continuity

* Transactions of this Society, vol. 41 (1937), pp. 84-123.

$\dagger$ The function $f(z)$ is said to satisfy a Lipschitz condition of order $\alpha$, $0<\alpha \leqq 1$, on the set $E$ if for $z_{1}$ and $z_{2}$ on $E$, we have $\left|f\left(z_{1}\right)-f\left(z_{2}\right)\right| \leqq L\left|z_{1}-z_{2}\right|^{\alpha}$, where $L$ is a constant independent of $z_{1}$ and $z_{2}$.

$\ddagger$ This Bulletin, vol. 42 (1936), pp. 873-878. 
properties of $f(z)$ on $E$ is called Problem $\alpha$, and the study of the relation between $\epsilon_{n}$ and the continuity properties of $f(z)$ on $C_{R}$ is called* Problem $\beta$. Thus in Theorem I we have a result on Problem $\alpha$ and in Theorem II a result on Problem $\beta$.

The method of proof of Theorems I and II is an application of conformal mapping to de la Vallée Poussin's † results on trigonometric approximation.

2. Proof of Theorem I. The analyticity of $f(z)$ interior to $C$ and the continuity of $f(z)$ in $\bar{C}$ follow directly from inequality (1). We consider first $C$ to be the unit circle: $|z|=1$. Let $f\left(e^{i \theta}\right) \equiv u(\theta)+i v(\theta)$; then inequality (1) on $C$ implies

$$
\left|u(\theta)-p_{n}(\theta)\right| \leqq \frac{M}{n^{p+\alpha}}, \quad\left|v(\theta)-q_{n}(\theta)\right| \leqq \frac{M}{n^{p+\alpha}},
$$

where $P_{n}\left(e^{i \theta}\right) \equiv p_{n}(\theta)+i q_{n}(\theta)$. But $p_{n}(\theta)$ and $q_{n}(\theta)$ are trigonometric sums of order $n$ and hence (de la Vallée Poussin, loc. cit., pp. 57 and 61-62) we have

$$
\begin{aligned}
& \left|u^{(p)}\left(\theta_{1}\right)-u^{(p)}\left(\theta_{2}\right)\right| \leqq L_{1}\left|\theta_{1}-\theta_{2}\right|^{\alpha}|\log | \theta_{1}-\theta_{2}|| \beta, \\
& \left|v^{(p)}\left(\theta_{1}\right)-v^{(p)}\left(\theta_{2}\right)\right| \leqq L_{2}\left|\theta_{1}-\theta_{2}\right|^{\alpha}|\log | \theta_{1}-\theta_{2}||^{\beta},
\end{aligned}
$$

where $\beta=0$ if $\alpha<1$ and $\beta=1$ if $\alpha=1$. Here $u^{(p)}(\theta)$ denotes the $p$ th derivative of $u(\theta)$ with respect to $\theta ; u^{(0)}(\theta) \equiv u(\theta)$. We have

$$
\begin{aligned}
& f^{\prime}\left(e^{i \theta}\right)=\frac{d f\left(e^{i \theta}\right)}{d e^{i \theta}}=\frac{d}{d \theta}(u(\theta)+i v(\theta)) \cdot \frac{d \theta}{d e^{i \theta}}=\left(u^{\prime}(\theta)+i v^{\prime}(\theta)\right) \frac{1}{i e^{i \theta}}, \\
& f^{\prime \prime}\left(e^{i \theta}\right)=\frac{d f^{\prime}\left(e^{i \theta}\right)}{d e^{i \theta}}=-\frac{1}{i e^{2 i \theta}}\left[i\left(u^{\prime \prime}(\theta)+i v^{\prime \prime}(\theta)\right)+\left(u^{\prime}(\theta)+i v^{\prime}(\theta)\right)\right],
\end{aligned}
$$

and similarly for higher derivatives.

Now $u^{(k)}(\theta), k=0,1,2, \cdots, p-1$, satisfies a Lipschitz condition of order 1 , as does the function $e^{-k i \theta}$. Thus we have

$$
\left|f^{(p)}\left(e^{i \theta_{1}}\right)-f^{(p)}\left(e^{i \theta_{2}}\right)\right| \leqq L_{3}\left|\theta_{1}-\theta_{2}\right|^{\alpha}|\log | \theta_{1}-\theta_{2} \mid{ }^{\beta}
$$

which through the properties of $e^{i \theta}$ implies inequality (2) for $z$ on $C$; the proof of Theorem $\mathrm{I}$ is complete for $C$ the unit circle.

* A more general formulation of these problems is given by Sewell, loc. cit., along with an extensive bibliography.

$\dagger$ Ch. J. de la Vallée Poussin, Lȩ̧ons sur l'Approximation des Fonctions d'une Variable Réele, Paris, 1919; see especially Chap. IV. 
Now let $C$ be an arbitrary analytic Jordan curve, and let the function $w=\Phi(z), z=\Psi(w)$, map $\bar{C}$ conformally onto $|w| \leqq 1$; the function $\Psi(w)$ is analytic and univalent in some closed region $|w| \leqq R_{0}>1$. Inequality (1) implies

$$
\left|f[\Psi(w)]-P_{n}[\Psi(w)]\right| \leqq \frac{M}{n^{p+\alpha}}, \quad|w| \leqq 1 .
$$

The polynomials $P_{n}(z)$ are uniformly bounded on $C$ for all $n$, say $\left|P_{n}(z)\right| \leqq M_{1}$; then by a well known theorem* we have (notation of Theorem II) $\left|P_{n}(z)\right| \leqq M_{1} R^{n}$ for $z$ on $C_{R}$. Let $\Gamma_{R_{1}}$ be the curve $|\Phi(z)|=R_{1}<R_{0}, R_{1}>1$, in the $z$-plane. Choose $R>1$ so that $\Gamma_{R_{1}}$ lies interior to $C_{R}$. Then $\left|P_{n}[\Psi(w)]\right| \leqq M_{1} R^{n}$ for $|w| \leqq R_{1}$. We have

$$
\begin{aligned}
P_{n}[\Psi(w)]-Q_{m}(w)=\frac{1}{2 \pi i} \int_{|t|=R_{1}} \frac{P_{n}[\Psi(t)] w^{m+1} d t}{(t-w) t^{m+1}}, \\
|w|<R_{1}<R_{0},
\end{aligned}
$$

where $Q_{m}(w)$ is the sum of the first $m+1$ terms of the Taylor development of $P_{n}[\Psi(w)]$ about the origin. This yields

$$
\left|P_{n}[\Psi(w)]-Q_{m}(w)\right| \leqq \frac{M_{1} R^{n} r^{m+1}}{\left(R_{1}-r\right) R_{1}{ }^{m}}, \quad|w| \leqq r<R_{\mathbf{1}} .
$$

Let us choose $r, 1<r<R_{1}$, and choose (this method is similar to that used by Curtiss, loc. cit.) $m=q n$ where $q$ is a positive integer such that $R\left(r^{q} / R_{1}^{q}\right)=r_{1}<1$; then we have

$$
\left|P_{n}[\Psi(w)]-Q_{q n}(w)\right| \leqq \frac{M_{1} r}{R_{1}-r} r_{1}^{n}, \quad|w| \leqq r>1 .
$$

Inequalities (7) and (8) yield

$$
\left|f[\Psi(w)]-Q_{q n}(w)\right| \leqq \frac{M}{n^{p+\alpha}}+\frac{M_{1} r}{R_{1}-r} r_{1}^{n}, \quad|w| \leqq 1 ;
$$

but we have $r_{1}<1$, and $\left(M_{1} r\right) /\left(R_{1}-r\right)$ is a constant independent of $n$, so for suitably chosen $M_{2}$ we have

$$
\left|f[\Psi(w)]-Q_{q n}(w)\right| \leqq \frac{M_{2}}{n^{p+\alpha}}=\frac{M_{2} q^{p+\alpha}}{(q n)^{p+\alpha}}, \quad|w| \leqq 1 .
$$

* See, for example, J. L. Walsh, Interpolation and Approximation, Colloquium Publications of this Society, vol. 20 (1935), pp. 77-78. 
Now choose $L_{N}(w) \equiv 0, N=1,2, \cdots, q-1, L_{q N^{\prime}+h}(w) \equiv Q_{q N^{\prime}}(w)$, $h=0,1,2, \cdots, q-1, N^{\prime}=1,2, \cdots$. Thus we have

$$
\left|f[\Psi(w)]-L_{N}(w)\right| \leqq \frac{M_{3}}{N^{p+\alpha}}, \quad|w| \leqq 1, N=1,2, \cdots,
$$

since when $M_{3}$ is suitably chosen we have $M_{2} q^{p+\alpha} / N_{1}^{p+\alpha}$ $\leqq M_{3} /\left(N_{1}+h\right)^{p+\alpha}, 0 \leqq h \leqq q-1$. Hence inequality (2) is satisfied by $d^{p} f[\Psi(w)] / d w^{p}$ in the one-dimensional sense on $|w|=1$. Since $\Psi(w)$ is analytic on $|w|=1$ it follows by the method used above* that inequality (2) is valid for $z$ on $C$; the proof of Theorem I is complete.

For the function $f(w)=\sum_{k=1}^{\infty} w^{k} / k(k-1)$ we have $(|w| \leqq 1)$

$$
\begin{aligned}
\left|f(w)-\sum_{k=1}^{n} \frac{w^{k}}{k(k-1)}\right| \leqq \sum_{k=n+1}^{\infty} \frac{1}{k(k-1)} & =\sum_{k=n+1}^{\infty}\left(\frac{1}{k-1}-\frac{1}{k}\right)=\frac{1}{n}, \quad n=1,2, \cdots ;
\end{aligned}
$$

but $f(w)$ does not satisfy a Lipschitz condition of order 1 on $|w|=1$ since the derivative $f^{\prime}(w) \equiv-\log (1-w)$ becomes infinite as $w$ approaches 1 . Thus for $\alpha=1$ the hypothesis of Theorem I does not imply a Lipschitz condition of order $\alpha$; an exact converse of Curtiss's theorem for $\alpha=1$ is impossible.

3. Proof of Theorem II. Let us set

$$
f(z)=P_{1}(z)+\left[P_{2}(z)-P_{1}(z)\right]+\left[P_{3}(z)-P_{2}(z)\right]+\cdots .
$$

Inequality (3) implies

$$
\begin{aligned}
& \left|P_{n+1}(z)-P_{n}(z)\right| \leqq \frac{2 M}{n^{p+\alpha+1} R^{n}}, \quad z \text { in } E, \\
& \left|P_{n+1}(z)-P_{n}(z)\right| \leqq \frac{2 M R}{n^{p+\alpha+1}}, \quad z \text { in } \bar{C}_{R} .
\end{aligned}
$$

Thus we have from equation (9) even exterior to $E$

$$
\left|f(z)-P_{n}(z)\right| \leqq \frac{M^{\prime}}{n^{p+\alpha}}, \quad z \text { in } \bar{C}_{R} .
$$
117.

* Or W. E. Sewell, this Bulletin, vol. 41 (1935), pp. 111-117; especially p. 
Theorem II now follows from Theorem I.

If we set for $|w| \leqq R>1$

$$
f(w)=\sum_{k=1}^{\infty} \frac{w^{k}}{R^{k} k(k-1)},
$$

we have for $|w| \leqq 1$

$$
\begin{aligned}
\mid f(w) & -\sum_{k=1}^{n} \frac{w^{k}}{R^{k} k(k-1)} \mid \leqq \sum_{k=n+1}^{\infty} \frac{1}{R^{k} k(k-1)} \\
& <\frac{1}{n(n+1)} \sum_{k=n+1}^{\infty} \frac{1}{R^{k}}<\frac{1}{R-1} \frac{1}{n^{2} R^{n}} .
\end{aligned}
$$

Yet $f(w)$ does not satisfy a Lipschitz condition of order 1 on $|w|=R$ since, as above, the derivative $f^{\prime}(w)$ becomes infinite as $w$ approaches $R$. As in $\S 2$ we consequently see that for $\alpha=1$ inequality (3) does not imply a Lipschitz condition of order $\alpha$.

4. Continuity of the Derivative in $\bar{C}$. To establish further properties of the functions $f(z)$ of Theorems I and II, and to relate these theorems to the results of Curtiss, we prove a third theorem:

THEOREM III. Let $C$ be an analytic Jordan curve, let the function $f(z)$ be analytic interior to $C$ and continuous in the corresponding closed region $\bar{C}$, and let the pth derivative $f^{(p)}(z)$ exist and be continuous on $C$ in the one-dimensional sense. Then $f^{(\boldsymbol{p})}(z)$ defined interior to $C$ in the usual two-dimensional sense and defined on $C$ in the one-dimensional sense is continuous throughout $\bar{C}$.

It is sufficient to prove the theorem for the case $p=1$, for the proof extends automatically by induction.

Let $C$ be the unit circle; the more general case can be transformed by conformal mapping to this special case. The original hypothesis fulfilled for an analytic curve $C$ implies the corresponding hypothesis for the unit circle, and the conclusion proved for the case of the unit circle implies the more general conclusion stated in the theorem.

The function $f(z)$ then satisfies the conditions

$$
\int_{C} f(z) z^{k} d z=0, \quad k=0,1,2, \cdots,
$$


by Cauchy's integral theorem. If on $C$ we set $z=e^{i \theta}, d z=i e^{i \theta} d \theta$, the Fourier development of $f(z)$ on $C$ by (10) can be written

$$
\begin{aligned}
f(z) & \sim \sum_{n=0}^{\infty} a_{n} e^{i n \theta}=\sum_{n=0}^{\infty} a_{n} z^{n}, \\
a_{n} & =\frac{1}{2 \pi} \int_{C} f(z) e^{-i n \theta} d \theta=\frac{1}{2 \pi i} \int_{C} \frac{f(z)}{z^{n+1}} d z .
\end{aligned}
$$

By partial integration we may write, for $k=0,1,-1,2,-2$, $\cdots$,

$$
\int_{C} f^{\prime}(z) z^{k} d z=\left[z^{k} f(z)\right]_{C}-k \int_{C} f(z) z^{k-1} d z .
$$

The first term on the right vanishes because $f(z)$ is single-valued and continuous on $C$. The Fourier development on $C$ of $f^{\prime}(z)$ is therefore by (10) and (12)

$$
f^{\prime}(z) \sim \sum_{n=1}^{\infty} n a_{n} e^{i(n-1) \theta}=\sum_{n=1}^{\infty} n a_{n} z^{n-1},
$$

where the $a_{n}$ are given by (11).

In (11) and (13) the series represent thus far only formal developments on $C$. But by the continuity of $f(z)$ in $\bar{C}$, the series $\sum_{n=0}^{\infty} a_{n} z^{n}$ is precisely the Taylor development of $f(z)$ valid throughout the interior of $C$. By differentiation it follows that $\sum_{n=1}^{\infty} n a_{n} z^{n-1}$ is the Taylor development of $f^{\prime}(z)$, likewise valid throughout the interior of $C$; it follows by inspection that this series is formally identical with the Fourier development (13) on $C$ of the derivative $f^{\prime}(z)$. By the continuity of $f^{\prime}(z)$ on $C$, the second form of the development (13) is valid uniformly on $C$ when summed by the method of arithmetic means; the corresponding sequence converges uniformly in $\bar{C}$ to the function $f^{\prime}(z)$ on $C$ and to the function $f^{\prime}(z)$ interior to $C$. Consequently the function $f^{\prime}(z)$ is continuous throughout $\bar{C}$, and the proof is complete.

Various refinements of Theorem III exist and are to be published by the present writers on another occasion.

HARVARD UNIVERSITY

Georgia School of Technology 